\title{
A Neutral Gas Model for Electron Swarms
}

\author{
Stefano L. Paveri-Fontana ${ }^{1,2}$ C. V. M. van der Mee, ${ }^{3,4}$ and P. F. Zweifel ${ }^{3}$
}

Received July 26, 1988; revision received June2, 1989

\begin{abstract}
A BGK-type Boltzmann equation for a neutral gas is considered as a model for electron swarms, because the gas and the electron Boltzmann equation have a common diffusion approximation. Both full- and half-range theory are developed using orthogonality methods of solution. Preliminary comparisons with diffusion theory are presented.
\end{abstract}

KEY WORDS: Diffusion; electron swarms; dispersion function.

\section{INTRODUCTION}

The motivation for this research comes from several studies ${ }^{(1.3)}$ on the influence of boundaries upon the behavior of a particle swarm, i.e., of a dilute population of charged particles diffusing through a neutral host gas under the influence of a dc space-uniform electric field $\mathbf{E}$ (see also, for example, the reviews in refs. 4 and 5). When ionization and recombination processes are in equilibrium, the pertinent linearized Boltzmann equation reads

$$
\left(\frac{\partial}{\partial t}+\mathbf{v} \cdot \frac{\partial}{\partial \mathbf{r}}+\mathbf{a} \cdot \frac{\partial}{\partial \mathbf{v}}\right) f(\mathbf{r}, \mathbf{v}, t)=[J f](\mathbf{r}, \mathbf{v}, t)
$$

Here $t, \mathbf{v}, \mathbf{r}, f$, and $J$ denote the time variable, the position vector, the velocity vector, the distribution function of the guest particles, and the

\footnotetext{
"Dipartimento di Metodi e Modelli Matematici, Università di Roma "La Sapienza," I-00161 Rome, Italy.

${ }^{2}$ Present address: Dipartimento di Matematica, Universitá di Milano, I-20133 Milan, Italy.

${ }^{3}$ Center for Transport Theory and Mathematical Physics, Virginia Polytechnic Institute and State University, Blacksburg, Virginia 24061.

${ }^{4}$ Present address: Department of Mathematical Sciences, University of Delaware, Newark, Delaware, 19716.
} 
collision term, respectively. In addition, for the acceleration vector we have $\mathbf{a}=q \mathbf{E} / m$, where $q$ is the charge and $m$ is the mass of each guest particle.

In the absence of a runaway effect, ${ }^{(6)}$ a simple macroscopic approximation for the process is the convection-diffusion equation

$$
\left(\frac{\partial}{\partial t}+\mathbf{w} \cdot \frac{\partial}{\partial \mathbf{r}}\right) c(\mathbf{r}, t)=D \nabla^{2} c(\mathbf{r}, t)
$$

where $c(\mathbf{r}, t):=\int_{\mathbb{R}^{3}} f(\mathbf{r}, \mathbf{v}, t) d \mathbf{v}$ is the concentration of the charged particles, $D$ is the diffusion coefficient, and $\mathbf{w}= \pm \mu \mathrm{E}$ is the drift velocity due to the dc electric field. Here $\mu$ is the mobility of the particles (ref. 5, p. 324) and the \pm sign corresponds to the sign of their charge.

Under steady-state conditions, when there is spatial slab symmetry and velocity azimuthal symmetry with respect to the direction of the acceleration field a, Eq. (1.1) can be rewritten in terms of one spatial variable (the component of $\mathbf{r}$ in the a direction) and two velocity variables (the components of $\mathbf{v}$ parallel and orthogonal to $\mathbf{a}$ ). A (rather questionable) approximation consists in dropping the dependence on the orthogonal component of the velocity $\mathbf{v}$. The resulting approximate steady-state version of Eq. (1.1) reads

$$
v \frac{\partial}{\partial x} f(x, v)+a \frac{\partial}{\partial v} f(x, v)=[J f](x, v)
$$

and is comparable with the one-dimensional $\mathrm{Kac}$ model $^{(7)}$ in nonlinear Boltzmann theory.

Equation (1.3a) appears to constitute a reasonable starting point for the study of some classes of boundary effects. Of course, a specific choice of the collision term $J$ must be made. One may adopt, for instance, the $\mathrm{BGK}^{(8)}$ relaxation time model:

$$
[J f](x, v)=-v_{0} f(x, v)+v_{0} m(v) \int_{-\infty}^{\infty} f(x, s) d s
$$

where $m(v)=(\beta / \pi)^{1 / 2} \exp \left(-\beta v^{2}\right)$ is the Maxwellian at the temperature of the background gas. This problem has been tackled by approximate methods. An exact explicit solution would be useful, since it would provide some insight into the physical mechanisms and constitute a "benchmark" against which to test the approximate solutions and Monte Carlo simulations. Specifically, it would allow a test of the reliability of the diffusion approximation. However, even in the simplified form (1.3) the problem turns out to be quite intricate. The application, for instance, of Case's singular eigenfunction technique ${ }^{(9)}$ turns out to be extremely difficult, since 
the spectrum of the pertinent operator occupies an entire half-plane. (However, it may be possible to apply the technique described by Cercignani. $\left.{ }^{(10)}\right)$

Now let us consider a different physical situation: that of a dilute gas of neutral particles diffusing within a moving background gas in the absence of an external electric field. It is easy to show that the diffusionlike equation for the population of guest particles is once again Eq. (1.2); however, now $\mathbf{w}$ is the convective velocity of the background gas. The counterparts of Eqs. (1.3) read

$$
\begin{aligned}
v \frac{\partial}{\partial x} f(x, v) & =-v_{0} f(x, v)+v_{0} \eta(v) \int_{-\infty}^{\infty} f(x, s) d s \\
\eta(v) & =\left(\frac{\beta}{\pi}\right)^{1 / 2} e^{-\beta(v-w)^{2}}
\end{aligned}
$$

Here $x$ is the component of $\mathbf{x}$ in the $\pm \mathbf{w}$ direction, $\eta$ is a drifting Maxwellian, and $\pm w$ is the magnitude of $\mathbf{w}$. Thus, the two formulations (1.3) and (1.4) describe two physical situations but share a common diffusion approximation, which in the absence of sources reads

$$
w \frac{\partial}{\partial x} c(x)=D \frac{\partial^{2}}{\partial x^{2}} c(x)
$$

In this paper we present a preliminary study of problem (1.4). The specific boundary conditions chosen will be suggested mainly by charged particle problems; we expect that our work will provide some insight into the harder problem (1.3) and also that it will allow some benchmark checks on Monte Carlo codes. We expect to be able to present specific asymptotic estimates and numerical in future work. This would allow us to test the reliability of the diffusion approximation. We expect to encounter some difficulties, since the spectrum of the linearized Boltzmann operator, unlike the spectrum of the diffusion operator, does not contain eigenvalues. The results in this paper generalize those for the case $w=0$ obtain earlier. ${ }^{(11)}$

In Section 2 we study the (singular) eigenvalues and eigenfunctions associated with Eq. (1.4) in detail. The result will be a continuous spectrum on the real line and, for $w \neq 0$, a simple eigenvalue at infinite. The latter is a departure from our experience for the conservative neutron transport equation and the linearized BGK model, which exhibit a double eigenvalue at infinity. ${ }^{(8,9,11)}$ In Sections 3 and 5 we obtain the full- and half-range eigenvalue expansions associated with Eq. (1.4), in analogy with the results of refs. 8, 9, and 11. These expansions are applied to an infinite-medium problem where charged particles are emitted by a permeable grid at $x=0$ 
(Section 4), and to the half-space problem with incoming flux boundary conditions (Section 6). The qualitative results for the half-space problem (with an electron-absorbing boundary) turn out to depend only on whether the convection process tends to move particles away from the boundary $(w>0)$ or back toward the boundary $(w<0)$. The latter case requires one to specify the asymptotic distribution [i.e., $f(+\infty, v)$ ] as an extra boundary condition.

\section{FORMULATION OF THE PROBLEM}

The introdifferential equation which we consider will be written in the dimensionless form

$$
v \frac{\partial f}{\partial x}(x, v)+f(x, v)=\eta(v \mid w) \int_{-\infty}^{\infty} f(x, s) d s, \quad v \in \mathbb{R}
$$

with

$$
\eta(v \mid w):=\frac{1}{\sqrt{\pi}} e^{-(v-w)^{2}}
$$

In these units both the thermal speed $\left(2 \kappa_{\mathrm{B}} T / m\right)^{1 / 2}$ and the collision frequency $v_{0}$ are equal to unity. The dimensionless drift velocity $w \in \mathbb{R}$ is assigned.

Following Case ${ }^{(19)}$ and Cercignani ${ }^{(8,11)}$ we seek elementrary solutions of the form

$$
f(x, v)=\varphi_{v}(v) e^{-x / v}
$$

subject to the usual normalization

$$
\int_{-\infty}^{\infty} \varphi_{v}(v) d v=1
$$

A standard treatment ${ }^{(8,9,11)}$ yields the discrete eigenmodes

$$
\varphi_{k}(v)=\frac{v_{k} \eta(v \mid w)}{v_{k}-v}
$$

where the eigenvalues are zeros of the dispersion function

$$
\Lambda(v \mid w):=1-\int_{-\infty}^{\infty} \frac{v}{v-s} \eta(s \mid w) d s=-\int_{-\infty}^{\infty} \frac{s}{v-s} \eta(s \mid w) d s, \quad v \in \mathbb{C} \backslash \mathbb{R}
$$


Hence

$$
\Lambda(v \mid w)=1+\frac{v}{\sqrt{\pi}} \int_{-\infty}^{\infty} \frac{e^{-t^{2}}}{t-(v-w)} d t, \quad v \in \mathbb{C} \backslash \mathbb{R}
$$

In terms of the tabulated ${ }^{(12)}$ Fried and Conte plasma dispersion function $Z$; we have

$$
A(v \mid w)=1+v Z(v-w), \quad \operatorname{Im} v>0
$$

We note the following useful relations:

$$
\begin{aligned}
\Lambda(-v \mid-w) & =\Lambda(v \mid w) \\
\Lambda(\bar{v} \mid w) & =\overline{\Lambda(v \mid w)}
\end{aligned}
$$

which hold for $v \in \mathbb{C} \backslash \mathbb{R}$ and $w \in \mathbb{R}$. Furthermore, for $u \in \mathbb{R}$ the Plemelj formulas imply

$$
A^{ \pm}(u \mid w):=\lim _{\varepsilon \downarrow 0} A(u \pm i \varepsilon \mid w)=\hat{\lambda}(u \mid w) \pm i \pi u \eta(u \mid w)
$$

where

$$
\begin{aligned}
\lambda(u \mid w):=1-\mathscr{P} \int_{-\infty}^{\infty} \frac{u}{u-s} \eta(s \mid w) d s & =\mathscr{P} \int_{-\infty}^{\infty} \frac{s}{s-u} \eta(s \mid w) d s \\
& =\frac{1}{2}\left[A^{+}(u \mid w)+\Lambda^{-}(u \mid w)\right]
\end{aligned}
$$

and

$$
\Lambda^{+}(0 \mid w)=\Lambda^{-}(0 \mid w)=1
$$

One can prove the following result.

Proposition 1. $A(\cdot \mid w)$ has no zeros on $\mathbb{C} \backslash \mathbb{R}$. Further, its limits $A^{ \pm}(\cdot \mid w)$ do not have real zeros.

In order to have an eigenvalue at infinity, Eq. (2.1) must have a nontrivial solution not depending on $x$ [cf. (2.3)]. From Eq. (2.1) we have immediately

$$
\varphi_{0}(v)=\eta(v \mid w)
$$

which satisfies the normalization $\int_{-\infty}^{\infty} \varphi_{0}(v) d v=1$. Generalized eigenvectors at $v=\infty$ correspond to solutions of Eq. (2.1) which are polynomials in $x$. It can be shown by straightforward calculation that there exist no such generalized eigenvectors if $w \neq 0$. In fact, we have the following result. 
Proposition 2. As $\quad v \rightarrow \pm \infty, \quad A(v \mid w) \sim-w / v$ for $w \neq 0$ and $\Lambda(v \mid w) \sim-1 / 2 v^{2}$ for $w=0$. ref. 12.

Proof. The proof follows directly from results of Section II of

From the above it is clear that $\varphi_{0}(v)=\eta(v \mid w)$ is the only discrete mode. [If $w=0$, one must also account for the generalized discrete mode $(x-v) \eta(v \mid 0)$.] In addition, we have for each $s \in \mathbb{R}$ the singular eigenfunction

$$
\varphi_{s}(v)=\eta(v \mid w) \mathscr{P} \frac{s}{s-v}+\lambda(s \mid w) \delta(v-s)
$$

which is in agreement with the normalization (2.4), provided $\lambda$ obeys (2.9b)

Direct study of $A(v \mid w)$ shows that if we introduce the continuous functions

$$
\Theta(s \mid w):=\arg \left(\Lambda^{+}(s \mid w)\right), \quad s \in \mathbb{R}
$$

we have, as $s \rightarrow+\infty$,

$$
\begin{aligned}
& \Theta(s \mid w)-\Theta(0 \mid w)=\pi+O\left(s^{2} e^{-s^{2}}\right), \quad w>0 \\
& \Theta(s \mid w)-\Theta(0 \mid w)=O\left(s^{2} e^{-s^{2}}\right), \quad w<0
\end{aligned}
$$

Similarly, we find for $w \neq 0$

$$
\Theta(+\infty \mid w)-\Theta(-\infty \mid w)=\pi \operatorname{sgn}(w)
$$

Formulas (2.11) and (2.12) will play a crucial role in the subsequent discussion of the full- and half-range expansions.

\section{FULL-RANGE EXPANSION}

To construct the solutions to infinite-medium problems, we shall be led to the solution of a singular integral equation of the form

$$
g(v)=\int_{-\infty}^{\infty} B(s) \varphi_{s}(v) d s, \quad v \in \mathbb{R}
$$

with

$$
g(v)=f(v)-a_{0} \varphi_{0}(v)
$$


Here $v f(v)$ is a uniformly Hölder continuous function subject to $\int_{-\infty}^{\infty}|v||f(v)| d v<+\infty, a_{0}$ is a discrete expansion coefficient, and $\varphi_{0}(v)=$ $\eta(v \mid w)$ is the discrete mode at infinity. If $w=0$, we add a term $a_{1} v \eta(v \mid 0)$ to the right-hand side of Eq. (3.1b), in order to account for the generalized eigenfunction $v \eta(v \mid 0)$. As this leads to a well-known result, ${ }^{(8,11)}$ we will refrain from considering this case in detail.

Using Eq. (2.11a) in (3.1a) gives

$$
g(v)=\lambda(v \mid w) B(v)+\eta(v \mid w) \mathscr{P} \int_{-\infty}^{\infty} B(s) \frac{s}{s-v} d s, \quad v \in \mathbb{R}
$$

We shall solve this equation by the orthogonality method. ${ }^{13)}$ First, we transform (3.2) to canonical form by defining

$$
h(v)=\frac{g(v)}{\eta(v \mid w)}, \quad A(s)=\frac{B(s)}{\eta(s \mid w)}
$$

in which case $(3.2)$ becomes

$$
h(v)=\hat{\lambda}(v \mid w) A(v)+\mathscr{P} \int_{-\infty}^{\infty} s A(s) \frac{\eta(s \mid w)}{s-v} d s, \quad v \in \mathbb{R}
$$

The solution is ${ }^{(13)}$

$$
B(s)=\frac{\lambda(s \mid w) g(s)}{A^{+}(s \mid w) A^{-}(s \mid w)}+\frac{\eta(s \mid w)}{X^{+}(s \mid w) A^{-}(s \mid w)} \mathscr{P} \int_{-\infty}^{\infty} \frac{X^{+}(v \mid w) g(v)}{A^{+}(v \mid w) s-v} d v
$$

Here the function $X(z \mid w)$ must be chosen according to the ratio condition

$$
\frac{X^{+}(v \mid w)}{X^{-}(v \mid w)}=\frac{A^{+}(v \mid w)}{\Lambda^{-}(v \mid w)}
$$

such that $X(z \mid w)^{-1}$ is bounded at infinity and analytic on $\mathbb{C} \backslash \mathbb{R}$, except for finitely many poles in $\mathbb{C} \backslash \mathbb{R} .^{(13)}$ An obvious choice for $X(z \mid w)$ is

$$
X(z \mid w)=(z-c) A(z \mid w)
$$

for some $c \in \mathbb{C} \backslash \mathbb{R}$. Since $X(z \mid w)$ has a pole at $z=c$, a constraint on $g(v)$ is introduced, ${ }^{(13)}$ which reduces to

$$
\int_{-\infty}^{\infty} t^{\prime} g\left(t^{\prime}\right) d t^{\prime}=0
$$


independent of c. As a result, we get, after using using (3.6) and (3.8) in (3.5),

$$
B(s)=\frac{\lambda(s \mid w) f(s)}{A^{+}(s \mid w) A^{-}(s \mid w)}+\frac{\eta(s \mid w)}{\Lambda^{+}(s \mid w) A^{-}(s \mid w)} \mathscr{P} \int_{-\infty}^{\infty} \frac{v f(v)}{s-v} d v, \quad s \in \mathbb{R}
$$

Recalling (3.1b), we see that $a_{0}$ can always be chosen such that $(3.8)$ is satisfied. Indeed,

$$
a_{0}=\frac{\int_{-\infty}^{\infty} v f(v) d v}{\int_{-\infty}^{\infty} v \varphi_{0}(v) d v}=\frac{1}{w} \int_{-\infty}^{\infty} v f(v) d v
$$

The fact that $B(s)$ is independent of $a_{0}$ is a consequence of orthogonality.

The summarize the results of this section in the following proposition.

Proposition 3. Let $f(v)$ be uniformly Hölder continuous on $\mathbb{R}$, let $\int_{-\infty}^{\infty}|v||f(v)| d v$ be finite, and let $w \neq 0$. Then there exist a constant $a_{0}$ and a function $B(s)$ such that

$$
f(v)=a_{0} \eta(v \mid w)+\int_{-\infty}^{\infty} B(s) \varphi_{s}(v) d s, \quad v \in \mathbb{R}
$$

with $B(s)$ given by (3.9) and $a_{0}$ by (3.10).

\section{AN INFINITE-MEDIUM PROBLEM}

The model problem we have in mind involves a grid at $x=0$ emitting electrons with the velocity distribution $q(v)$ in both directions. The grid is assumed permeable both to the host particles and the electrons. (The effect of the imposed electric field is modeled by a drift velocity $w$ in the opposite direction.) Thus, we seek solutions to the homogeneous transport equation (2.1) subject to the "jump condition"

$$
f\left(0^{+}, v\right)-f\left(0^{-}, v\right)=\frac{q(v)}{v}
$$

It is assumed that $q(v) \geqslant 0, v \in \mathbb{R}$; that $q(v) / v$ is uniformly Hölder continuous on $\mathbb{R}$; and finally, that the source strength is finite, namely

$$
Q:=\int_{-\infty}^{\infty} q(v) d v<+\infty
$$


Moreover, without loss of generality we assume that $w>0$. The grid is suppose to be the unique source of electrons. Thus we supplement (4.1a) by the upstream condition

$$
f(-\infty, v)=0
$$

and we require $f(x, v)$ to remain bounded as $x \rightarrow+\infty$. Then the solution is given by

$$
f(x, v) \begin{cases}a_{0} \eta(v \mid w)+\int_{0}^{\infty} B(s) \varphi_{s}(v) e^{-x / s} d s, & x>0 \\ -\int_{-\infty}^{o} B(s) \varphi_{s}(v) e^{-x / s} d s, & x<0\end{cases}
$$

The jump condition gives

$$
\frac{q(v)}{v}=a_{0} \eta(v \mid w)+\int_{-\infty}^{\infty} B(s) \varphi_{s}(v) d s
$$

with $a_{0}$ and $B(v)$ given by (3.9) and (3.10) with $f(v)=q(v) / v$. In particular, we have

$$
a_{0}=\frac{1}{w} Q
$$

so that asymptotically for large $x$ we have

$$
f(+\infty, v)=Q \eta(v \mid w) / w
$$

Moreover,

$$
B(s)=\frac{\lambda(s \mid w) q(s)}{s \Lambda^{+}(s \mid w) \Lambda^{-}(s \mid w)}+\frac{\eta(s \mid w)}{\Lambda^{+}(s \mid w) \Lambda^{-}(s \mid w)} \mathscr{P} \int_{-\infty}^{\infty} \frac{q(v)}{s-v} d v
$$

which can be written, with the help of $(2.9 \mathrm{~b})$, as

$$
B(s)=\frac{1}{s \Lambda^{+}(s \mid w) \Lambda^{-}(s \mid w)} \int_{-\infty}^{\infty} \frac{v q(s) \eta(v \mid w)-s q(v) \eta(s \mid w)}{v-s} d v
$$

Note that the integral is not a principal value integral. The electron concentration is given by

$$
c(x)= \begin{cases}Q / w+\int_{0}^{\infty} B(s) e^{-x / s} d s, & x>0 \\ -\int_{-\infty}^{0} B(s) e^{-x / s} d s, & x<0\end{cases}
$$

Conversely, the solution of this problem under the diffusion approximation, i.e., the pertinent solution of Eq. (1.5), reads

$$
c(x)= \begin{cases}Q / w, & x>0 \\ (Q / w) e^{w x / D}, & x<0\end{cases}
$$


provided

$$
Q_{1}:=\int_{-\infty}^{\infty} v q(v) d v=0
$$

The remainder of this section is devoted to a comparison of expressions (4.8) and (4.9). We see that the two expressions are in satisfactory agreement if and only if (i) $B(s)$ is sharply peaked in the neighborhood of $-D / w$; (ii) it is negligible elsewhere; and finally (iii) we have $c\left(0^{+}\right)=$ $c\left(0^{-}\right)$, i.e.,

$$
\int_{-\infty}^{\infty} B(s) d s=-Q / w
$$

Now, with the help of Eqs. (2.4), (4.4), and (4.5) we see that (4.11) holds if and only if

$$
\int_{-\infty}^{\infty} \frac{q(v)}{v} d v=0
$$

which is to be compared with (4.10). Of course, both (4.10) and (4.12) are valid if $q(v)$ is even.

In order to study the position of the peaks of $B(s)$, let us now consider expression (4.7a). Since the expression in the denominator

$$
\Lambda^{+}(s \mid w) \Lambda^{-}(s \mid w)=\lambda(s \mid w)^{2}+\pi^{2} s^{2} \eta(s \mid w)^{2}
$$

is strictly positive for $s \in \mathbb{R}$ and $q(v) / v$ is Hölder continuous, we can claim that $B(s)$ is not singular at $s=0$. It may exhibit peaks in the vicinity of the (possible) peaks of the source function $q(v)$ as well as in the vicinity of the minimum points of $A^{+} A^{-}$. We shall concentrate our attention on the latter putative peak points.

First, we recall that $\eta(s \mid w)=\pi^{-1 / 2} \exp \left[-(s-w)^{2}\right]>0$ for all $s \in \mathbb{R}$ and we observe that, consistent with Eqs. (2.7) and (2.9b),

$$
\lambda(s \mid w)=1-2 s F(s-w), \quad s \in \mathbb{R}
$$

where

$$
F(x):=e^{-x^{2}} \int_{0}^{x} e^{t^{2}} d t
$$

is Dawson's integral. ${ }^{(14)}$ Then we introduce

$$
y(x):=\frac{1}{F(x)}-2 x
$$

and remark that $\lambda$ has a zero at $s=x+w \in \mathbb{R}$ if and only if $y(x)=2 w$. The function $y(x)$ can be studied with the help of Table 7.5 of ref. 14. We find 
that, as $x$ increases from 0 toward $+\infty, y(x)$ first descends from $+\infty$ toward its minimum at $y\left(x_{b}\right) \simeq-0.72$ at $x_{b} \simeq 1.75$ and then increases toward $y(+\infty)=0^{-}$. The unique positive zero of $y(x)$ is at $x_{a} \simeq 0.92$. The behavior of $y(x)$ for negative $x$ can be established by reflection, since $y(x)$ is an odd function. At this point we can claim that, if $0<w<-y\left(x_{b}\right) / 2$, then $\lambda(s \mid w)$ has three zeros: $s_{0}(w) \in\left(-\infty,-x_{b}+w\right), s_{1}(w) \in\left(-x_{b}+w\right.$, $\left.-x_{a}+w\right)$, and $s_{2}(w) \in\left(w, x_{a}+w\right)$. On the other hand, if $w>-y\left(x_{b}\right) / 2$, then $\lambda$ has a unique zero which is in the interval $\left(w, x_{a}+w\right)$. Now, a zero $s_{k}$ of $\lambda(s \mid w)$ may be expected to correspond to a sharp peak of $\left(\Lambda^{+} \Lambda^{-}\right)^{-1}$ only if $s_{k} \eta\left(s_{k} \mid w\right)$ is small; i.e., only if $\left|s_{k}-w\right| \gg 1$. This occurs for only one of the three zeros listed above, $s_{0}(w)$, and only when $w$ is small compared to one. Indeed, since ${ }^{(12)}$

$$
y(x)=\frac{1}{F(x)}-2 x=-\frac{1}{x}\left[1+\frac{1}{x^{2}}+O\left(\frac{1}{x^{4}}\right)\right] \quad \text { as } \quad x \rightarrow \pm \infty
$$

we see that $y(x)-2 w$ has a large zero at

$$
x_{0}=-\frac{1}{2 w}\left[1+4 w^{2}+O\left(w^{4}\right)\right] \quad \text { as } \quad w \rightarrow 0^{+}
$$

which corresponds to

$$
s_{0}=x_{0}+w=-\frac{1}{2 w}\left[1+2 w^{2}+O\left(w^{4}\right)\right] \quad \text { as } \quad w \rightarrow 0^{+}
$$

where $\lambda\left(s_{0} \mid w\right)=0$. For the derivative we have

$$
\lambda^{\prime}\left(s_{0} \mid w\right)=-\frac{1+2 s_{0} w}{s_{0}}=-4 w^{3}\left[1+O\left(w^{2}\right)\right]=\frac{1}{2 s_{0}^{3}}\left[1+O\left(\frac{1}{s_{0}^{2}}\right)\right]
$$

Accordingly, when $s \simeq s_{0}$ in the $w \rightarrow 0^{+}$limit (i.e., as $s_{0} \rightarrow-\infty$ ),

$$
\begin{aligned}
& \lambda(s) \frac{q(s)}{s}+\eta(s \mid w) \mathscr{P} \int_{-\infty}^{\infty} \frac{q(v)}{s-v} d v \\
& \simeq \frac{\eta(s \mid w)}{s} \int_{-\infty}^{\infty} q(v)\left(1+\frac{v}{s}+\frac{v^{2}}{s^{2}}+\cdots\right) d v \\
& \simeq \frac{\eta\left(s_{0} \mid w\right)}{s_{0}} Q\left[1+O\left(\frac{1}{s_{0}}\right)\right] \\
& A^{+}(s \mid w) A^{-}(s \mid w) \\
& \simeq\left(s-s_{0}\right)^{2} \lambda^{\prime}\left(s_{0} \mid w\right)^{2}+\pi^{2} s^{2} \eta(s \mid w)^{2} \\
& \simeq \frac{\left(s-s_{0}\right)^{2}}{4 s_{0}^{6}}+\pi^{2} s_{0}^{2} \eta\left(s_{0} \mid w\right)^{2}
\end{aligned}
$$


so that Eq. (4.7a) yields

$$
-B(s) \simeq \frac{2 \pi s_{0}^{4} \eta\left(s_{0} \mid w\right)}{\left(s-s_{0}\right)^{2}+\left(2 \pi s_{0}^{4}\right)^{2} \eta\left(s_{0} \mid w\right)^{2}} \frac{2\left|s_{0}\right| Q}{\pi}
$$

The integration of the expression on the right-hand side of (4.15) with respect to $s$ on $\mathbb{R}$ gives $2\left|s_{0}\right| Q$.

We can draw the conclusion that, when $0<w \ll 1,-B(s)$ does indeed exhibit a peak, which is extremely sharp, since its (approximate) width and height are

$$
\Delta s \simeq 2 \pi s_{0}^{4} \eta\left(s_{0} \mid w\right) \simeq 2 \pi^{1 / 2} s_{0}^{4} \exp \left(-s_{0}^{2}\right)
$$

and

$$
-B\left(s_{0}\right) \simeq \frac{Q}{\pi^{3 / 2}} \frac{\exp \left(s_{0}^{2}\right)}{\left|s_{0}\right|^{3}}
$$

respectively. The center is (approximately) at $s_{0} \simeq-1 /(2 w) \ll-1$. The area is approximately $2\left|s_{0}\right| Q \simeq Q / w$, to be compared with (4.11). Accordingly, we can claim that if the drift speed is small compared to the thermal speed $(0<w \ll 1)$, then there is good agreement between the diffusion theory expansion (4.9) and the transport theory result (4.8), provided we set [in agreement with (4.14)]

$$
D=-s_{0} w=\frac{1}{2}+w^{2}+O\left(w^{4}\right)
$$

Agreement between (4.8) and (4.9) is clearly not as good when the convective velocity is comparable (or large with respect) to the speed of thermal agitation. However, introducing the Fourier transform of the concentration

$$
\hat{c}(k)=\int_{-\infty}^{\infty} e^{-i k x} c(x) d x, \quad k \in \mathbb{R}
$$

one can show that

$$
\hat{c}(k) i k(w-i k D)=Q
$$

according to diffusion theory, whereas

$$
\hat{c}(k) \int_{-\infty}^{\infty} \frac{i k v}{1+i k v} \eta(v \mid w) d v=\int_{-\infty}^{\infty} \frac{q(v)}{1+i k v} d v
$$


according to transport theory. Expanding in powers of $k$ the latter result, we have

$$
\begin{array}{r}
\hat{c}(k) \int_{-\infty}^{\infty} i k v\left[1-i k v+O\left(k^{2} v^{2}\right)\right] \eta(v \mid w) d v \\
\quad=\int_{-\infty}^{\infty} i k v\left[1-i k v+O\left(k^{2} v^{2}\right)\right] q(v) d v
\end{array}
$$

Since

$$
\begin{aligned}
& \int_{-\infty}^{\infty} v \eta(v \mid w) d v=w \\
& \int_{-\infty}^{\infty} v^{2} \eta(v \mid w) d v=\frac{1}{2}+w^{2}
\end{aligned}
$$

we can write $(4.18)$ as follows

$$
\hat{c}(k) i k\left[w-i k\left(\frac{1}{2}+w^{2}\right)+O\left(w^{2}\right)\right]=Q\left[1+O\left(k^{2}\right)\right]
$$

provided (4.10) holds and $\int_{-\infty}^{\infty} v^{2} q(v) d v<+\infty$. Comparison of (4.17) with (4.20) shows that there is agreement (to lower orders in powers of $k$ ) between the two expressions (originating from diffusion theory and transport theory, respectively), provided that one sets

$$
D=\frac{1}{2}+w^{2}=\int_{-\infty}^{\infty} v^{2} \eta(v \mid w) d v
$$

This result, which is consistent with (4.16), is somewhat surprising. One may assume that the diffusion of a dilute gas of guest particles within a drifting host medium ought to be governed by the latter's thermal agitation (i.e., the average kinetic energy of the host molecules as seen by a Lagrangian observer moving with the host gas); in this case, we would have found $D=\int_{-\infty}^{\infty}(v-w)^{2} \eta(v \mid w) d v$. On the contrary, (4.21) indicates that the diffusion process is governed by the thermal agitation of the host gas as seen by an observer who does not move with the convective host gas.

\section{HALF-RANGE EXPANSION}

For solving problems on a half-space, we shall be led to solving singular integral equations of the form

$$
g(v)=\int_{0}^{\infty} B(s) \varphi_{s}(v) d s, \quad v>0
$$


Transforming it to the canonical form as in Section 3, we obtain

$$
h(v)=\lambda(v) A(v)+\int_{0}^{\infty} s A(s) \frac{\eta(s \mid w)}{s-v} d s
$$

where $h(v)=g(v) / \eta(v \mid w)$ and $A(s)=B(s) / \eta(s \mid w)$. The solution is then given by

$$
B(s)=\frac{\lambda(s \mid w) g(s)}{A^{+}(s \mid w) \Lambda^{-}(s \mid w)}+\frac{\eta(s \mid w)}{X^{+}(s \mid w) \Lambda^{-}(s \mid w)} \mathscr{P} \int_{0}^{\infty} v \frac{X^{+}(v \mid w)}{\Lambda^{+}(v \mid w)} \frac{g(v)}{s-v} d v
$$

In this case, however, the $X$ function satisfies a different ratio condition from (3.6) because of the different range of integration. Specifically,

$$
\frac{X^{+}(s \mid w)}{X^{-}(s \mid w)}=\frac{A^{+}(s \mid w)}{A^{-}(s \mid w)}, \quad s>0
$$

but

$$
X^{+}(s \mid w)=X^{-}\left(\left.s\right|^{\prime} w\right), \quad s \leqslant 0
$$

The fundamental solution $X_{0}(z \mid w)$ is well known ${ }^{(9,15)}$ and has then form

$$
X_{0}(z \mid w)=\exp \left(\frac{1}{\pi} \int_{0}^{\infty} \frac{\theta(s \mid w)}{s-z} d s\right), \quad \arg (z) \in(0,2 \pi)
$$

where $\theta(s \mid w)=\arg A^{+}(s \mid w)$. The analysis of ref. 13 indicates that (5.2) satisfies Eq. (5.1b) if and only if $X(z \mid w)^{-1}$ is bounded at infinity and

$$
X(z \mid w)=O\left(z^{\alpha}\right) \quad \text { as } \quad z \rightarrow 0
$$

for some $0 \leqslant \alpha<1$. Certainly, $X_{0}(z \mid w)$ is bounded, while

$$
X_{0}(z \mid w) \sim z^{-\theta(0 \mid w) / \pi} \quad \text { as } \quad z \rightarrow 0
$$

provided $\int_{0}^{\infty} \theta(s \mid w) d s$ is finite. Referring to Eqs. (2.11), we see that this integral is finite for $w<0$ and diverges for $w>0$.

Consider the case $w<0$ first. We note that $\theta(0 \mid w)=0$, so condition (5.5) is satisfied and the solution is given by (5.2) with $X=X_{0}$.

For $w>0$ it is necessary to renormalize $X_{0}(z \mid w)$ to ${ }^{(13)}$

$$
X_{1}(z \mid w)=\exp \left(\frac{1}{\pi} \int_{0}^{\infty} \frac{\theta(s \mid w)-\pi}{s-z} d s\right), \quad \arg (z) \in(0,2 \pi)
$$


Then $X_{1}(z \mid w)$ still obeys the ratio condition, but now $\int_{0}^{\infty}[\theta(s \mid w)-\pi] d s$ is finite. We see that $X_{1}(z \mid w)$ is bounded at infinity, while

$$
X_{1}(z \mid w) \sim z \quad \text { as } \quad z \rightarrow 0
$$

Thus we are led to choose for $X(z \mid w)$

$$
X_{2}(z \mid w)=\frac{z-c}{z} X_{1}(z \mid w)
$$

for some $x \in \mathbb{C} \backslash \mathbb{R}$. The subsequent analysis is similar to the one of Section 3, involving a constraint induced by the pole at $z=c$. Again, the constraint is satisfied by choosing $g(v)=f(v)-a_{0} \eta(v \mid w)$. We find rather easily

$$
\begin{aligned}
a_{0} & =\frac{\int_{0}^{\infty} f(v) X_{1}^{+}(v \mid w) A^{+}(v \mid w)^{-1} d v}{\int_{0}^{\infty} \eta(v \mid w) X_{1}^{+}(v \mid w) A^{+}(v \mid w)^{-1} d v} \\
& =-\int_{0}^{\infty} f(v) X_{1}^{+}(v \mid w) A^{+}(v \mid w)^{-1} d v
\end{aligned}
$$

since the identity

$$
\eta(v \mid w) X_{1}^{+}(v \mid w) A^{+}(v \mid w)^{-1}=\frac{1}{2 \pi i}\left[X_{1}^{+}(v \mid w)-X_{1}^{-}(v \mid w)\right]
$$

followed by some straightforward contour integration can be used to show that

$$
\int_{0}^{\infty} \eta(v \mid w) X_{1}^{+}(v \mid w) A^{+}(v \mid w)^{-1} d v=-1
$$

The continuum coefficients reduce to

$$
\begin{array}{r}
B(s)=\frac{\lambda(s \mid w) f(s)}{\Lambda^{+}(s \mid w) \Lambda^{-}(s \mid w)}+\frac{s \eta(s \mid w)}{X_{1}^{+}(s \mid w) \Lambda^{-}(s \mid w)} \mathscr{P} \int_{0}^{\infty} \frac{X_{1}^{+}(v \mid w)}{\Lambda^{+}(v \mid w)} \frac{f(v)}{s-v} d v \\
s>0
\end{array}
$$

Note that $X_{1}$, and not $X_{2}$, enters the expressions for $a_{0}$ and $B(s)$, which are, of course, independent of $c$.

We collect the results of this section as a proposition.

Proposition 4. Let $f(v)$ be uniformly Hölder continuous on $[0, \infty)$ and let $\int_{0}^{\infty}|f(v)| d v$ be finite. Then: 
(a) For $w>0$ there exist a constant $a_{0}$ and a function $B(s)$ such that

$$
f(v)=a_{0} \eta(v \mid w)+\int_{0}^{\infty} B(s) \varphi_{s}(v) d s, \quad v>0
$$

Here $a_{0}$ and $B(s)$ are given by (5.8) and (5.10), respectively.

(b) For $w<0$ there exists a function $B(s)$ such that

$$
f(v)=\int_{0}^{\infty} B(s) \varphi_{s}(v) d s, \quad v>0
$$

Here $B(s)$ is given by (5.2) [setting $X=X_{0}$, Eq. (5.4), and $\left.g=f\right]$.

\section{HALF-SPACE PROBLEMS}

We consider the half-space $x>0$ with the particles injected at $x=0$. We consider separately the case in which the convection process drives the particles away from the boundary $(w>0)$ and the case in which the convection process drives the particles back toward the boundary $(w<0)$. The case $w>0$ would correspond to an electron transport problem in which electrons are released from a cathode (at $x=0$ ) with a velocity distribution $f_{0}(v), v>0$, and accelerated in an electric field in the positive $x$ direction. Moreover, those electrons which are driven back to the cathode by collision are assumed to be absorbed. Conversely, the case $w<0$ corresponds to electrons being driven by an electric field in the negative $x$ direction toward an anode located at $x=0$. The incoming electron current is assumed to be given. In addition, electrons may evaporate from the anode into the $x>0$ semispace with a distribution $f_{0}(v)$. In either case, $w>0$ or $w<0$, we require the distribution to be bounded as $x \rightarrow+\infty$.

Case 1. $w>0$. By virtue of the above discussion, we see that we must impose the condition

$$
f(0, v)=f_{0}(v), \quad v>0
$$

Then Proposition 4 implies that

$$
f(x, v)=a_{0} \eta(v \mid w)+\int_{0}^{\infty} B(s) e^{-x / s} \varphi_{s}(v) d s, \quad x>0, \quad v \in \mathbb{R}
$$

with $a_{0}$ and $B(s)$ given by Eqs. (5.8) and (5.10) and the downstream distribution by

$$
f(+\infty, v)=a_{0} \eta(v \mid w)
$$


The entering current at $x=0$, the outgoing current at $x=0$, and the downstream net current are given by the respective expressions

$$
\begin{aligned}
J_{+}(0) & =\int_{0}^{\infty} v f(0, v) d v \\
J_{-}(0) & =\int_{0}^{\infty}|v| f(0,-v) d v \\
J_{\infty} & =\int_{-\infty}^{\infty} v f(+\infty, v) d v=a_{0} w
\end{aligned}
$$

We have by particle conservation

$$
J_{+}(0)=J_{-}(0)+J_{\infty}
$$

Then the transmission $T$ is given by

$$
T=J(+\infty) / J_{+}(0)=-w \int_{0}^{\infty} X_{1}^{+}(v \mid w) A^{+}(v \mid w)^{-1} f_{0}(v) d v / \int_{0}^{\infty} v f_{0}(v) d v
$$

and the albedo is given by $1-T$.

Case 2. $w<0$. The previous discussion indicates that, in addition to (6.1), we must impose the condition

$$
f(+\infty, v)=\eta(v \mid w) q, \quad \text { with } \quad q \geqslant 0
$$

Then the solution is given by

$$
f(x, v)=q \eta(v \mid w)+\int_{0}^{\infty} B(s) \varphi_{s}(v) e^{-x / s} d s, \quad x>0, \quad s \in \mathbb{R}
$$

We compute $B(s)$ from Eq. (5.10) with $g(v)$ replaced by $f_{0}(v)-q \eta(v \mid w)$. Thus,

$$
B(s)=B_{0}(s)-q B_{\eta}(s)
$$

where $B_{0}$ and $B_{\eta}$ are computed from (5.10) with data $f_{0}$ and $\eta$, respectively. In fact, $B_{\eta}$ can be computed explicitly by a contour integration similar to one used in ref. 13. The result is

$$
B_{\eta}(s)=\frac{\eta(s \mid w)}{X_{1}^{+}(s) \Lambda^{-}(s)}
$$

We note the similarity of this case to the Milne problem ${ }^{(9)}$ and the Kramers problem, ${ }^{(8,11)}$ in which a source at infinity is assumed. 


\section{DISCUSSION}

The problem discussed here is a one-dimensional "caricature" of an electron transport problem. In this model, convection and scattering govern the equations of motion of electrons, whereas in the true physical situation it is the electric field which together with the scattering process drives the electrons. When the electron distribution function has a velocity profile which is not far from a Maxwellian (at the temperature and the drift velocity of the background gas), we may expect the two models to yield results which are close to each other, and not far from the results of the corresponding diffusion approximation, provided drift velocity, acceleration, diffusion coefficient, etc., are properly matched.

An indication that this model may have some merit stems from the qualitative agreement between the results of transport theory and those from diffusion theory (cf. discussion in Section 4). Further comparison between expression (6.6) for the transmission coefficient and its counterpart according to diffusion theory-at an asymptotic or a numerical level-may be of interest.

Finally, we remark that, although Eq. (1.4) looks very much like the evaporation model studied by Arthur and Cercignani, ${ }^{(16)}$ the qualitative results depend essentially on whether $w$ exceeds the speed of sound of the evaporizing gas or not, whereas in the present problem the sign of $w$ is all that matters.

The mathematical model dealt with in this paper is very simple. More general transport equations have been discussed in recent monographs. ${ }^{(17,18)}$ It is possible that the methods discussed in these monographs could be utilized to deal with more realistic, and hence more complicated, models of the transport process we have attempted to describe in this paper. This question is still being studied.

\section{ACKNOWLEDGMENTS}

The research described here was supported by the U.S. Department of Energy grant DE-FG-05-87ER25033 and National Science Foundation grant DMS 8701050. Two of the authors (CvdM and SLPF) would lilke to thank the Center for Transport Theory and Mathematical Physics and the Department of Physics of Virginia Tech for their hospitality during visits in 1987-1988 when the work described here was carried out. SLPF worked on this project under the auspices of GNFM-CNR (Italy) and of the "Equations of Evolution" MPI (Italy) project. 


\section{REFERENCES}

1. R. E. Robson, Aust. J. Phys. 28:523-531 (1975).

2. R. E. Robson, Aust. J. Phys. 29:171-175 (1976).

3. R. E. Robson, Aust. J. Phys. 34:323-341 (1981).

4. K. Kumar, H. R. Skullerud, and R. E. Robson, Austr. J. Phys. 33:343-448 (1980).

5. K. Kumar, Phys. Rep. 112:319-375 (1984).

6. G. Cavalleri and S. L. Paveri-Fontana, Phys. Rev. A 6:327-333 (1972).

7. M. Kač, Phys. Fluids 2:8-12 (1959).

8. C. Cercignani, Theory and Application of the Boltzmann Equation (Elsevier, New York, 1975); The Boltzmann Equation and its Applications (Springer, Berlin, 1988).

9. K. M. Case and P. F. Zweifel, Linear Transport Theory (Addison-Wesley, Reading, Massachusetts, 1967).

10. C. Cercignani, Ann. Phys. (N.Y.) 40:454-468 (1966).

11. C. Cercignani, Ann. Phys. (N.Y.) 20:219-233 (1962).

12. B. D. Fried and S. D. Conte, The Plasma Dispersion Function. The Hilbert Transform of the Gaussian (Academic Press, New York, 1961).

13. C. V. M. van der Mee and P. F. Zweifel, Applications of orthogonality relations to singular integral equrations, J. Int. Eqs. Appl., to appear.

14. M. Abramowitz and I. A. Stegun, Handbook of Mathematical Functions (Dover, New York, 1964).

15. N. I. Muskhelishvili, Singular Integral Equations (Noordhoff, Groningen, 1953).

16. M. D. Arthur and C. Cercignani, Z. Angew. Math. Phys. 31:634-645 (1980).

17. H. G. Kaper, C. G. Lekkerkerker, and J. Hejtmanek, Spectral Methods in Linear Transport Theory (Birkhäuser, Basel, 1982).

18. W. Greenberg, C. V. M. van der Mee, and V. Protopopescu, Boundary Value Problems in Abstract Kinetic Theory (Birkhäuser, Basel, 1987). 